\title{
Van Der Knaap Disease in a 3-year-old Male Child: A Case Report
}

\author{
Imran Mahmood Khan ${ }^{1}$, Asma Shabbir ${ }^{2}$, Sadaf $\mathrm{Naz}^{3}$, Rubina Zulfqar ${ }^{4}$ \\ ${ }^{1}$ Associate Professor, Pediatric Department, Islamabad Medical and Dental College, Islamabad Pakistan \\ ${ }^{2}$ Associate Professor, Pediatric Department, Fazaia Medical College, Islamabad, Pakistan \\ ${ }^{3}$ Consultant Radiologist, Radiology Department, Islamabad Diagnostic Center, Islamabad, Pakistan \\ ${ }^{4}$ Professor, Pediatric Department, Islamabad Medical and Dental College, Islamabad, Pakistan
}

\begin{abstract}
Van der Knaap disease or megalencephalic leukoencephalopathy with subcortical cysts is a leukodystrophy with autosomal-recessive inheritance caused by mutation in the gene MLC1 which is localized on chromosome 22q. It is characterized by macrocephaly, motor developmental delay, seizures, spasticity, ataxia, and mild mental deterioration. On neuroimaging, involvement of cerebral white matter along with subcortical cysts in frontal and temporal lobes are hallmarks of the disease. There is no definite treatment of this disease. We report a case of Van Der Knaap disease in a 3-year-old male child who presented with seizures and delayed developmental milestones.

Key Words: Degenerative brain disease, Leukodystrophy, Macrocephaly, Megalencephaly, Subcortical cysts, Van der Knaap

Correspondence:

Imran Mahmood Khan

Article info:

Email: lifesaverforu@yahoo.com

Received: April 18, 2020

Cite this case report: Khan IM, Shab

Cite this case report: Khan IM, Shabbir A, Naz S, Zulfaar R. Van Der Knaap Disease in a 3-year-

old Male Child: A Case Report. J Islamabad Med Dental Coll. 2020; 9(2):.145-148. Doi: 10.35787/jimdc.v9i2.537

(16, 2020

Funding Source: Nil

Conflict of Interest: Nil

disease
\end{abstract}

\section{Introduction}

Van der Knaap disease or megalencephalic leukoencephalopathy (MLC)with subcortical cysts is a leukodystrophy with autosomal-recessive inheritance. ${ }^{1}$ It is characterized by early-onset macrocephaly, motor developmental delay, seizures, spasticity, ataxia, and mild mental deterioration. ${ }^{2}$ This disease is present globally but occurs most commonly in the Aggarwal community of India with a high frequency of consanguineous marriages. ${ }^{3}$ Due to rarity of this disease, exact incidence is not known. So far, in Pakistan only one case has been reported in the literature. ${ }^{4}$ Here we report the case of a child with delayed developmental milestones, who was brought by his parents to Pediatric OPD with main complaint of a seizure one month back. He was finally diagnosed as a case of a very rare disease (Van der Knaap disease) with no specific treatment.

\section{Case Report}

A 3-year-old male child, resident of Neelam Valley, Azad Kashmir, Pakistan, presented in the OPD with complaints of nystagmus, seizures and delayed milestones. According to the parents, the child developed nystagmus at the age of 6 months. So far, he had only one episode of seizures one month back, which was generalized tonic clonic type, lasting for 2 to 3 minutes and stopped spontaneously. The child was not taking any antiepileptic drug. He was a product of nonconsanguineous marriage with one younger and two elder sisters. He was born at term through 
spontaneous vaginal delivery without any antenatal, natal or postnatal complications. His developmental milestones were delayed with neck holding achieved at 7 months of age and sitting at 12 months. He started walking at 2 years of age with history of frequent falls during walking. His vision, hearing, speech and cognition were appropriate for his age.

On physical examination, his weight was $11.2 \mathrm{~kg}$ and height were $85 \mathrm{~cm}$, both were less than 5 th percentile for his age. His anterior fontanelle was open and head circumference was $53 \mathrm{~cm}$ which was above 97th percentile. He had hypertonia and hyperreflexia in both lower limbs. He had no dysmorphic features or visceromegaly. Rest of the physical examination was unremarkable.

His baseline investigations like CBC, ESR, serum electrolytes, calcium and magnesium were normal. On the basis of large head, open anterior fontanelle and other symptoms, provisional diagnosis of hydrocephalous was made and CT scan brain was advised. However, CT scan brain showed significant diffuse hypodensity of the cerebral white matter, predominantly of the frontal, temporal and parietal lobes. This was associated with thinning of the cortex and bilateral temporal and left parietal subcortical cysts suggestive of megalencephalic leukoencephalopathy with subcortical cysts (Figure 1). The hallmarks of the disease were quite evident on CT brain, so it was decided that MRI brain the gold standard for diagnosis of Van der Knaap disease, was no more needed. Syrup sodium valproate was started for the control of seizures and multivitamins prescribed to increase his growth.

Parents were counselled that no specific treatment was available for this slowly progressive inherited disease with $25 \%$ chances of occurrence in every pregnancy. As they had no wish to have more children, they refused genetic testing. Follow-up visit after one month revealed a stable condition with no seizure during this period.

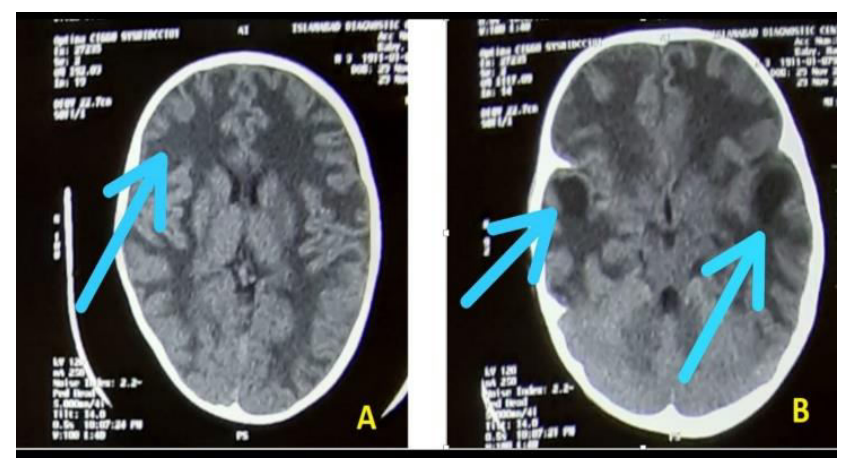

Figure 1A: CT scan image showing diffuse hypodensity of cerebral white matter predominantly of frontal, temporal and parietal lobes associated with thinning of the cortex as shown with arrow. 1B: CT scan image showing bilateral temporal subcortical cysts as shown with arrows.

\section{Discussion}

Megalencephalic leukoencephalopathy was first described by the Indian neurologist Bhim Sen Singhal in 1991 in the Agarwal community. ${ }^{5}$ Later in 1995, Dutch neurologist Marjo Van der Knaap described the series of cases in 8 children and the disease was referred to as Van der Knaap disease. ${ }^{6}$ It is characterized by macrocephaly, delayed milestones and seizures, which start in early infancy and has slow progressive neurodegenerative course. ${ }^{6}$ Seizures are easily controlled by common antiepileptic drugs. ${ }^{7}$ There are also extrapyramidal abnormalities with dystonia and athetosis along with spasticity and ataxia which usually develop by 5 years of age. Cognitive delay is milder and occurs later than motor delay. ${ }^{8}$

MLC result from defect in brain ion and water homeostasis resulting in chronic cerebral white matter edema and vacuole formation. ${ }^{9}$ It is an autosomal recessive disease resulting from mutation in MLC1 gene on chromosome 22q leading to an imbalanced intracellular ion concentration and astrogliosis. ${ }^{9}$ 
MRI brain is superior to CT brain due to its ability to depict anatomy in greater detail and hence can differentiate between various differential diagnoses. MRI of the brain in Van der Knaap disease show's increased characteristic diffuse supratentorial white matter signal intensity on T2weighted images, decreased signal intensity on T1weighted images with relative sparing of grey and deep white matter structures such as corpus collosum, internal capsule and brain stem. Cysts are present in the anterior temporal area and often in the frontoparietal area. ${ }^{10}$ Later in life, white matter swelling decreases and cerebral atrophy comes into the picture. $^{8}$

Main differential diagnoses are Alexander's disease, infantile-onset GM1 and GM2 gangliosidosis and Canavan's disease. All these diseases have rapidly progressive course with almost $100 \%$ mortality in early childhood or adolescence. In contrast, MLC has a milder course despite highly abnormal $\mathrm{CT} / \mathrm{MRI}$ findings and life expectancy is up to $3 \mathrm{rd}$ and 4 th decade of life. ${ }^{2,4}$

MRI in infantile GM1 and GM2 gangliosidosis and Canavan's disease shows involvement of the globus pallidus, basal ganglia and thalamus which are spared in MLC, in addition to the white matter abnormalities. In Alexander disease, white matter involvement is predominant in frontal regions and shows contrast enhancement which is usually absent in MLC., ${ }^{411}$

Currently there is no definite treatment of MLC, although trials of gene therapy are going on. ${ }^{12}$ Treatment is only symptomatic for complications in the form of antiepileptic drugs and physiotherapy. ${ }^{2,3}$ The best strategy is prevention of this disease in affected families by avoiding consanguineous marriages and providing prenatal diagnosis of future pregnancies through amniocentesis and chorionic villus sampling., ${ }^{4,11}$

\section{Conclusion}

MLC should be included in the differential diagnosis of macrocephaly and seizures. Imaging studies of brain are sufficient to make the diagnosis in the presence of particular symptoms. Genetic testing for prenatal diagnosis should be done to identify the particular mutations in future pregnancies. Affected families should be educated and counselled regarding risks of consanguineous marriages in order to decrease genetic diseases in the community.

\section{References}

1. López-Hernández $T$, Ridder $M C$, Montolio $M$, Capdevila-Nortes X, Polder E, Sirisi S, et al. Mutant Glial CAM causes megalencephalic leukoencephalopathy with subcortical cysts, benign familial macrocephaly, and macrocephaly with retardation and autism. Am J Hum Genet. 2011; 88(4): 422-32. Doi: 10.1016/j.ajhg.2011.02.009

2. Sardana V, Bhattiprolu RK. Van der knaap disease: A case report. Indian J Case Reports. 2018; 4(5): 3756.

3. Singhal BS. Leukodystrophies: Indian scenario. Indian J Pediatr. 2005; 72(4): 315-8. Doi: 10.1007/BF 02724013

4. Bokhari M, Inayat F, Sardar J, Bokhari R. Van der Knaap Disease. J Coll Physicians Surg Pakistan. 2018; 28(11): 888-90. Doi: 10.29271/jcpsp.2018.11.888

5. Singhal BS, Gursahani RD, Udani VP, Biniwale AA. Megalencephalic leukodystrophy in an Asian Indian ethnic group. Pediatr Neurol. 1996; 14(4): 291-6. Doi: 10.1016/0887-8994(96)00048-3

6. Van der Knaap MS, Barth PG, Stroink H, van Nieuwenhuizen O, Arts WFM, Hoogenraad F, et al. Leukoencephalopathy with swelling and $a$ discrepantly mild clinical course in eight children. Ann Neurol. 1995; 37(3): 324-34. Doi: 10.1002/ana. 410370308

7. Batla A, Pandey S, Nehru R. Megalencephalic leukoencephalopathy with subcortical cysts: A report of four cases. J Pediatr Neurosci. 2011; 6(1): 74-7. Doi: 10.4103/1817-1745.84416 
8. Dash PK, Raj DH, Sahu H. Megalencephalic leucoencephalopathy with subcortical cysts: subcortical diffuse leuco-encephalopathy associated with white matter cystic degeneration. BMJ Case Rep. 2015; bcr2015211921. Doi: 10.1136/bcr-2015211921

9. Van der Knaap MS, Boor I, Estévez R. Megalencephalic leukoencephalopathy with subcortical cysts: Chronic white matter oedema due to a defect in brain ion and water homoeostasis. Lancet Neurol. 2012; 11(11): 973-85. Doi: 10.1016/ S1474-4422(12)70192-8

10. Reis F, Yoshio R, Kido Z, Mesquita JA, Oshima MM, Montenegro MA. Megalencephalic leuko- encephalopathy with subcortical cysts a case with clinical and magnetic resonance imaging dissociation. Arq Neuropsiquiatr. 2015; 73(2): 1712. Doi: $10.1016 / \mathrm{S} 1474-4422$

11. Roy U, Joshi B, Ganguly G. Van der Knaap disease: a rare disease with atypical features. BMJ Case Rep. 2015; bcr2015209831. Doi:10.1136/bcr-2015209831

12. Van der Knaap MS, Schiffmann R, Mochel F, Wolf NI. Diagnosis, prognosis, and treatment of leukodystrophies. Lancet Neurol. 2019; 18(10): 96272. Doi: 10.1016/S1474-4422(19)30143-7 